\title{
Drug-induced psoriasis: clinical perspectives
}

This article was published in the following Dove Press journal:

Psoriasis:Targets and Therapy

\section{Deepak MW Balak \\ Enes Hajdarbegovic}

Department of Dermatology, Erasmus University Medical Center, Rotterdam, the Netherlands
Correspondence: Deepak MW Balak Department of Dermatology, Erasmus University Medical Center, Burgemeester S'jacobplein 5I, 3015 CA, Rotterdam, the Netherlands

Tel +3। $10703 \quad$ II87

Fax +3। 107033822

Email balak.dmw@gmail.com

\begin{abstract}
Exposure to certain drugs can elicit an induction or exacerbation of psoriasis. Although well-conducted systematic studies on drug-related psoriasis are mostly lacking, traditionally strong associations have been documented for beta-blockers, lithium, antimalarial drugs such as (hydroxy)chloroquine, interferons, imiquimod, and terbinafine. More recently, new associations have been reported for monoclonal antibody- and small-molecule-based targeted therapies used for oncological and immunological indications, such as tumor necrosis factor-alpha antagonists and anti-programmed cell death protein 1 immune checkpoint inhibitors. Recognizing potential drug-related psoriasis is of clinical relevance to allow an optimal management of psoriasis. However, in clinical practice, identifying medication-related exacerbations and induction of psoriasis can be challenging. The clinical and histopathological features of drug-provoked psoriasis may differ little from that of "classical" nondrug-related forms of psoriasis. In addition, the latency period between start of the medication and onset of psoriasis can be significantly long for some drugs. Assessment of the Naranjo adverse drug reaction probability scale could be used as a practical tool to better differentiate drug-related psoriasis. The first step in the management of drug-related psoriasis is cessation and replacement of the offending drug when deemed clinically possible. However, the induced psoriasis skin lesions may persist after treatment withdrawal. Additional skin-directed treatment options for drugrelated psoriasis follows the conventional psoriasis treatment guidelines and includes topical steroids and vitamin D analogs, ultraviolet phototherapy, systemic treatments, such as acitretin, methotrexate, and fumaric acid esters, and biological treatments.
\end{abstract}

Keywords: psoriasis, drug-induced, psoriasiform, cutaneous drug reaction, beta-blocker, lithium, monoclonal antibodies, small molecules

\section{Introduction}

Psoriasis is a chronic, immune-mediated inflammatory skin disease that is associated with a high physical and psychosocial burden of disease. ${ }^{1}$ With estimated prevalences reaching up to $11 \%$ in adults and up to $1.3 \%$ in children, psoriasis is a common disease. ${ }^{2}$ Consequently, psoriasis is frequently encountered in clinical practice, by general practitioners and dermatologists alike.

The etiology of psoriasis is multifaceted. A complex interplay between genetic predisposing factors and environmental factors leads to the onset of psoriasis. ${ }^{3}$ In addition, various triggering factors have been linked to an exacerbation of psoriasis, such as infection, physical trauma to the skin (ie, the Koebner phenomenon), and exposure to emotional stress. ${ }^{4}$

Another clinically important external factor that can elicit psoriasis is exposure to drugs. There are several ways in which a drug can affect psoriasis (Table 1). ${ }^{5-7}$ 
Table I Possible scenarios of drug-related psoriasis

Drug involvement in psoriasis
Exacerbation of psoriasis
Exacerbation of preexisting psoriasis $\rightarrow$ remission following treatment
discontinuation
Exacerbation of preexisting psoriasis $\rightarrow$ persisting following treatment
discontinuation
Induction of psoriasis
Induction of psoriasis on previously clinically uninvolved skin in a patient
with a personal history of psoriasis
Induction of psoriasis de novo in a patient without a personal of family
history of psoriasis

First, a drug can cause preexisting psoriatic skin lesions to aggravate. Upon discontinuation of the implicated drug, the psoriasis exacerbation can decrease (ie, drug-induced psoriasis). Alternatively, the induced psoriatic skin lesions can persist (ie, drug-aggravated psoriasis). Second, a drug can cause onset of new psoriasis lesions at clinically uninvolved skin in a patient with a personal history of psoriasis. Third, medication-use can provoke psoriasis de novo in a patient without a personal or family history of psoriasis.

Certain drugs have been linked strongly to psoriasis. Examples of these include beta-blockers, lithium, antimalarial drugs such as chloroquine, interferons, imiquimod, and terbinafine. ${ }^{5}$ In addition to these, new targeted treatments with monoclonal antibodies and small molecules used in oncology and immunology may induce psoriasis. Furthermore, rapid treatment withdrawal of systemic corticosteroids or potent topical corticosteroids may elicit a psoriasis flare (ie, rebound effect). ${ }^{8}$

Recognizing the potential role of medication in psoriasis is of clinical importance, considering that drug utilization is in general high among psoriasis patients. ${ }^{9}$ This may be due to the treatment of systemic comorbidities that are linked to psoriasis, such as hypertension and other cardiovascular diseases. Alternatively, surveillance bias may underlie the increased drug utilization among patients with psoriasis. ${ }^{10}$ Whatever the underlying reasons, the relatively high number of medications used by psoriasis patients makes potential associations between medication use and psoriasis exacerbation or induction relevant in clinical practice.

However, in daily clinical practice, there may be insufficient attention to potential involvement of medication-related causes for psoriasis induction, exacerbation, or treatmentresistance. ${ }^{11}$ Moreover, identifying medication-related exacerbations and induction of psoriasis can prove to be difficult in daily clinical practice for various reasons. First, the association between drugs and psoriasis onset and/or exacerbation is frequently not well-studied, resulting in a low level of strength in terms of evidence. Second, the lag time between start of the medication and onset is variable and can be quite lengthy. Third, in some cases, the psoriasis flare can persist, even after the suspected drug has been discontinued. As a result, the clinical identification of drug-related psoriasis can be challenging.

Taken together, recognizing potential drug involvement in psoriasis is of clinical relevance to allow an optimal management of psoriasis. ${ }^{5}$ This review is aimed at giving a comprehensive overview of the clinical presentation of drug-induced psoriasis.

\section{Methods}

This narrative review is based on a nonsystematic literature search in Medline and Embase databases. The search date was performed in January 2017 with an update of the search in June 2017. The following keywords were used: "psoria*" and "drug-induced". We included articles written in English reporting the development of psoriasis related to medication use either in children or in adults. There were no restrictions set in study type. We focused primarily on articles from the last decade. In addition, we included several reviews written on drug-induced psoriasis. ${ }^{5,6,12-15}$

\section{Results}

\section{Potential drug involvement in psoriasis}

In clinical practice, identifying drug-related causes of a psoriasis flare can be difficult for various reasons. One complicating factor is that the latency period between start of the medication and onset of psoriatic skin lesions can vary considerably between drugs. Medications with a typically short latency period include terbinafine, whereas lithium and beta-blockers can be associated with relatively long latency periods up to 12 months. ${ }^{11}$ Consequently, a temporal relationship is sometimes not immediate apparent. A psoriasis flare in the absence of known triggering factors such as infection and emotional stress should raise the possibility of a drug-related cause. Another issue is that the psoriasis flare can persist following discontinuation of the suspected medication. Moreover, there may be little difference between "classical" psoriasis and drug-related psoriasis in terms of clinical and histopathological features.

A tool to help to better discern potential causal drug relations is the Naranjo adverse drug reaction probability scale. ${ }^{13,16}$ The scale consists of 10 questions, the scoring of which results into four categories: definite, probable, possible, and doubtful (Table 2). Assessment with the Naranjo scale 
Table 2 The Naranjo adverse drug reaction probability scale

\begin{tabular}{|c|c|c|c|c|}
\hline \multirow[t]{2}{*}{ Question } & & \multicolumn{3}{|c|}{ Answer } \\
\hline & & Yes & No & Do not know \\
\hline $\mathrm{I}$ & Are there previous conclusive reports on this reaction? & +1 & 0 & 0 \\
\hline 2 & Did the adverse event appear after the suspected drug was administered? & +2 & -1 & 0 \\
\hline 3 & $\begin{array}{l}\text { Did the adverse event improve when the drug was discontinued or a specific antagonist was } \\
\text { administered? }\end{array}$ & +1 & 0 & 0 \\
\hline 4 & Did the adverse event reappear when the drug was readministered? & +2 & -1 & 0 \\
\hline 5 & Are there alternative causes that could on their own have caused the reaction? & -1 & +2 & 0 \\
\hline 6 & Did the reaction reappear when a placebo was given? & -1 & +1 & 0 \\
\hline 7 & Was the drug detected in blood or other fluids in concentrations known to be toxic? & +1 & 0 & 0 \\
\hline 8 & $\begin{array}{l}\text { Was the reaction more severe when the dose was increased or less severe when the dose } \\
\text { was decreased? }\end{array}$ & +1 & 0 & 0 \\
\hline 9 & $\begin{array}{l}\text { Did the patient have a similar reaction to the same or similar drugs in any previous } \\
\text { exposure? }\end{array}$ & +1 & 0 & 0 \\
\hline 10 & Was the adverse event confirmed by any objective evidence? & +1 & 0 & 0 \\
\hline Total score & Interpretation: probability of an adverse drug reaction & & & \\
\hline$\geq 9$ & Definite & & & \\
\hline $5-8$ & Probable & & & \\
\hline $1-4$ & Possible & & & \\
\hline$\leq 0$ & Doubtful & & & \\
\hline
\end{tabular}

Note: Reproduced with permission from Naranjo CA, Busto U, Sellers EM, et al. A method for estimating the probability of adverse drug reactions. Clin Pharmacol Ther 1981;30(2):239-245.16 @ 198I American Society for Clinical Pharmacology and Therapeutics.

could be helpful for clinical practice to strengthen a possible drug association.

Of note, the available evidence supporting the associations of drug-related psoriasis with various drugs is limited predominantly to anecdotal single-case reports or retrospective case series. Exceptions to this are beta-blockers, lithium, and tumor necrosis factor (TNF)-alpha antagonists, for which the associations with drug-related psoriasis were assessed in large-cohort studies or case-control studies.

\section{Pathophysiological mechanisms of drug- related psoriasis}

The underlying mechanisms leading to drug-induced or drugaggravated psoriasis are mostly incompletely understood. For some medications such as lithium, beta-blockers, and imiquimod, a direct drug effect is suspected. ${ }^{17,18}$ Alternatively, indirect mechanisms are sometimes implicated via a Koebner phenomenon, eg, in case of a drug hypersensitivity reaction, allergic reaction, irritant reaction, and phototoxicity. ${ }^{12,19}$

Several drugs have a well-described mode of action by which they can induce the development of psoriasis. Such mechanistic insights have helped to eludicate the pathophysiological pathways underlying psoriasis. One example is interferon-alpha, which plays an important role in the initiation of psoriasis. In psoriatic skin, activated plasmacytoid dendritic cells produce type I interferons, such as interferon-alpha, which in turn triggers a cascade leading to proinflammatory cytokine production. ${ }^{20}$ Similarly, exogenously administered interferon-alpha is able to induce Th1- and Th17-mediaded cutaneous inflammation. ${ }^{21}$

Closely related to the role of interferon-alpha in psoriasis development is imiquimod. Imiquimod is a topical toll-like receptor (TLR) 7 agonist that can activate cutaneous plasmacytoid dendritic cells to produce interferons. ${ }^{18}$ In psoriasis, self-nucleic acids bound to antimicrobial peptides such as LL37 can engage TLRs, which in turn initiate TLR-mediated proinflammatory responses, such as activation of plasmacytoid dendritic cells. ${ }^{3}$ In line, an imiquimod-induced psoriasis mouse model is used to study psoriasis. ${ }^{22}$

\section{Clinical features of drug-induced psoriasis}

The clinical spectrum of drug-related psoriasis is as diverse as "typical" nondrug-related psoriasis itself. ${ }^{5}$ Morphological types that have been described as drug reaction included plaque psoriatic skin lesions, palmoplantar psoriasis, nail psoriasis, scalp psoriasis leading to alopecia, pustular psoriasis, and erythrodermic psoriasis. Furthermore, transformative drug-reactions have been described, eg, pustular psoriasis induction in a patient with a personal history of plaque psoriasis. There are no clear specific psoriasis phenotypes provoked by the different drugs implicated in drug-related psoriasis.

There are no clear clinical criteria to distinguish the morphology of drug-induced psoriasis to that of typical psoriasis. However, in some cases, so-called psoriasiform skin lesions are described. ${ }^{23}$ Clinically, such skin lesions are almost similar to classic psoriasis cases but lack certain 
characteristics of psoriasis. For instance, psoriasiform skin lesions may miss well-demarcated borders or lack the coarse scaling typical of psoriasis.

The severity of drug-related psoriasis can vary considerable. There have been documented cases of generalized psoriasis and erythrodermic psoriasis, requiring hospitalization for proper management. ${ }^{19,24}$

\section{Histopathological features of drug- induced psoriasis}

There are few systematic studies on the histopathology of drug-induced psoriasis. In most cases, the histopathology of a drug-induced psoriasis is virtually indistinguishable from that of conventional psoriasis. However, drug-induced psoriasis often lacks tortuous papillary dermal capillaries and associated suprapapillary epidermal thinning, which may be used as differential criterion. ${ }^{25}$ Furthermore, the number of Munro micro-abscesses and macrophages may be low in drugrelated psoriasis. ${ }^{5}$ Additional histological clues to diagnose a potential drug-induced psoriasis include an eosinophilic infiltrate in the dermis and a lichenoid pattern. ${ }^{19,25,26}$ Importantly, however, absence of these histopathological findings does not exclude the possibility of a drug-related psoriasis.

\section{Management of drug-induced psoriasis}

The first step in the management of drug-related psoriasis is stopping and replacing the offending agent when possible. However, the psoriasis skin lesions may show no to minimal improvement upon treatment discontinuation of the suspected drug. When additional skin-directed therapy is needed, the treatment options that are normally available for psoriasis can be initiated. These options include topical treatments with corticosteroids and/or vitamin D analogs, ultraviolet-based phototherapy and systemic treatments, such as methotrexate, acitretin, and fumaric acid esters, and biologics. ${ }^{27}$ Cases have been reported in which systemic treatment with methotrexate or cyclosporine was needed to sufficiently control druginduced psoriasis. ${ }^{19,28}$

\section{Specific drug associations linked to psoriasis}

The list of drugs that have associations with drug-related psoriasis is quite extensive. For most drugs, however, the evidence is limited to anecdotal reports. Consequently, there are often insufficient grounds to clearly define the risk of a particular drug in context of its psoriasis induction of exacerbation potential. Traditionally, there have been strong associations documented for beta-blockers, lithium, synthetic antimalarial drugs, interferons, imiquimod, and terbinafine (Table 3). Of note, most of these associations stem from the 1970s and 1980s, in particular for lithium and synthetic antimalarial drugs.

\section{Traditionally reported drug- associations Beta-blockers}

Beta-blockers, also known as beta-adrenergic receptor antagonists, have been among the drugs most strongly linked to plaque psoriasis exacerbation. It is noted that the lag time to onset of psoriasis development can take up to 12 months. ${ }^{11}$ Oral beta-blocker use has been anecdotally implicated in erythrodermic psoriasis ${ }^{19,29}$ and palmoplantar pustular

Table 3 Overview of selected traditional and newly reported drug-associations linked to either induction or exacerbation of psoriasis

\begin{tabular}{|c|c|c|c|}
\hline \multirow[t]{2}{*}{ Drug } & \multirow[t]{2}{*}{ Indication } & \multicolumn{2}{|l|}{ Association } \\
\hline & & $\begin{array}{l}\text { Induction of } \\
\text { psoriasis }\end{array}$ & $\begin{array}{l}\text { Exacerbation } \\
\text { of psoriasis }\end{array}$ \\
\hline \multicolumn{4}{|l|}{ Traditional drug-associations } \\
\hline Beta-adrenergic receptor antagonists (beta-blockers) & Cardiovascular diseases, glaucoma, etc & $x$ & $x$ \\
\hline Lithium & Manic-depressive disorder & $x$ & $x$ \\
\hline Synthetic antimalarial drugs (eg, chloroquine) & Malaria, lupus erythematosus & $x$ & $x$ \\
\hline $\begin{array}{l}\text { Acute withdrawal of systemic corticosteroid or } \\
\text { potent topical corticosteroid }\end{array}$ & Dermatological indications & & $x$ \\
\hline Interferons (alpha, beta) & Hepatitis, certain malignancies, multiple sclerosis & $x$ & $x$ \\
\hline \multicolumn{4}{|l|}{ Novel drug-associations } \\
\hline Anti-tumor necrosis factor alpha antagonists & Immune-mediated inflammatory diseases & $x$ & \\
\hline Anti-PDI immune checkpoint inhibitors & Oncology & $x$ & $x$ \\
\hline Bupropion (= nicotine receptor antagonist) & Smoking cessation treatment & $x$ & $x$ \\
\hline VEGF antagonists & Oncology & $x$ & $x$ \\
\hline Rituximab (anti-CD20) & Oncology and immune-mediated inflammatory diseases & $x$ & $x$ \\
\hline
\end{tabular}


psoriasis. ${ }^{28}$ Topical beta-blocking agents, used for the treatment of glaucoma, have been reported to elicit psoriatic nail changes. ${ }^{30}$ In pediatric dermatology, oral beta-blocking agents are prescribed for the treatment of infantile hemangiomas. There is a single case of the development of psoriasiform diaper rash linked to treatment with oral propranolol in an 18-month-old girl. ${ }^{31}$ While there are convincing individual case reports on the association between beta-blocker use and psoriasis provocation, epidemiological studies yielded conflicting results. ${ }^{32} \mathrm{~A}$ large US prospective cohort study using data from the US Nurses' Health Study reported longterm regular beta-blocker use for $\geq 6$ years to be statistically significantly associated with an increased risk for psoriasis (HR, 1.39; 95\% CI, 1.11-1.73). ${ }^{33}$

\section{Lithium}

Lithium, which is prescribed for manic depressive disorders, has been associated with an increased risk for psoriasis development. The first report of psoriasis exacerbation linked to lithium use dates from the 1970 s. ${ }^{34}$ Cases on psoriasis induction and psoriasis exacerbation due to lithium have been reported, but lithium seems to cause predominantly exacerbations. ${ }^{5,7}$ Lithium-related psoriasis flares may respond favorable to oral supplementation with inositol. ${ }^{17}$ In a case-control study using UK-based general practice data, long-term lithium use was associated with an increased risk for incident psoriasis (odds ratio 1.68, 95\% CI 1.18-2.39). ${ }^{35}$ Psoriasis types linked to lithium include plaque type psoriasis, pustular psoriasis, palmoplantar psoriasis, and psoriatic arthritis. ${ }^{15,36}$ The mean time between start of lithium and onset of psoriasis exacerbations is $\sim 20$ weeks, while time to induction of psoriasis is estimated to be 48 weeks. ${ }^{11,15}$

\section{Antimalarial drugs: chloroquine and hydroxychloroquine}

The synthetic antimalarial drugs chloroquine and hydroxychloroquine are associated with plaque type psoriasis exacerbations. ${ }^{5}$ There have been several descriptions of worsening of psoriasis in individuals receiving antimalarial prophylaxis. ${ }^{37,38}$ Furthermore, there have been cases reported of psoriasis exacerbation with the use of hydroxychloroquine as immunomodulating treatment for lichen planopilaris and psoriatic arthritis. ${ }^{39}$ Rarely, these antimalarial drugs are linked to induction of psoriasis. ${ }^{40}$ The latency period of psoriasis exacerbation is usually between 4 and 12 weeks. ${ }^{11}$ Morphological variants of (hydroxy)-chloroquine-related psoriasis other than plaque type psoriasis include pustular psoriasis and erythroderma. ${ }^{41-43}$ The exact risk of psoriasis exacerbation or induction related to antimalarial drugs remains unknown. ${ }^{44}$

\section{Imiquimod}

The TLR7 agonist imiquimod is used as topical treatment for various indications including (pre)-malignant skin lesions. Imiquimod treatment can lead to psoriasis exacerbation in adult individuals already diagnosed with psoriasis. ${ }^{18}$ Imiquimod has been linked also to drug-related psoriasis in children, who had been treated off-label for molluscum contagiosum or verrucae ${ }^{45}$ Most cases describe plaque-type psoriasis as reaction to imiquimod. One case described the development of psoriatic erythroderma following topical imiquimod treatment for actinic keratosis; in this case, infliximab treatment was needed to achieve sufficient disease control. ${ }^{26}$

\section{Interferons}

Interferons have a well-known association with psoriasis development. Different interferons have been described in relation with induction of psoriasis, including interferonalpha, interferon-beta, and interferon-gamma.

Interferon-alpha treatment is used for multiple indications, including hepatitis $\mathrm{B}$ or $\mathrm{C}$ infection, Kaposi sarcoma, and various malignancies, such as renal cell carcinoma and metastatic melanoma. Psoriasis has been described predominantly in patients with hepatitis $\mathrm{C}^{21,46,47}$ but also infrequently in patients with melanoma, ${ }^{48}$ leukemia, ${ }^{49,50}$ Kaposi sarcoma, and renal cell carcinoma. ${ }^{51}$ Of note, both cutaneous and - more rarely - psoriatic arthritis have been linked to interferon-alpha treatment. ${ }^{51}$

Treatment with interferon-beta is indicated for multiple sclerosis. Both exacerbation and induction of psoriasis have been described in patients with multiple sclerosis treated with interferon-beta. ${ }^{52-54}$

Finally, interferon-gamma, which was studied as a potential psoriasis treatment in the early 1990s, has been described to induce psoriasis at the injection site of interferon-gamma. ${ }^{55,56}$

\section{Terbinafine}

Another drug that is commonly linked to an increased risk for psoriasis is terbinafine, a synthetic antifungal agent. ${ }^{57-59}$ Terbinafine is described in relation with both exacerbation and induction of psoriasis..$^{60}$ Morphological psoriasis variants triggered by terbinafine treatment include plaque type psoriasis, (generalized) pustular psoriasis, and inverse psoriasis. ${ }^{60-63}$ 


\section{Novel reported drug associations} Anti-TNF-alpha antagonists

Monoclonal antibody-based antagonists of TNF-alpha are in use for a variety of immune-mediated inflammatory diseases, such as Crohn's disease, rheumatoid arthritis, and psoriasis. Surprisingly, use of TNF-alpha inhibitors is associated with a risk of developing psoriasiform adverse events. The development of this so-called paradoxal psoriasis - given that TNF-alpha antagonists are applied as biological treatment for psoriasis - is well-documented for nondermatological indications of anti-TNF-alpha antagonism, such as inflammatory bowel disease and rheumatoid arthritis. Morphological psoriasis variants induced by TNF-alpha antagonists include plaque type psoriasis, pustular psoriasis, guttate psoriasis, scalp psoriasis, and palmoplantar psoriasis. ${ }^{64}$

A systematic review published in 2017 analyzed 216 cases of new-onset psoriasis induced by TNF-alpha inhibitor use for Crohn's disease ( $41 \%$ of cases), rheumatoid arthritis (37\%), and ankylosing spondylitis (14\%). ${ }^{65}$ The prevalence of anti-TNF-induced psoriasis in inflammatory bowel disease is $\sim 2 \% .{ }^{66}$ The majority of cases had onset of psoriasis development within the first year of treatment with the TNF-alpha inhibitor. Female sex and smoking were more frequently associated with TNF-alpha inhibitor-induced psoriasis. ${ }^{66,67} \mathrm{~A}$ previous retrospective cohort study in inflammatory bowel disease patients also reported smoking as an important predictor for anti-TNF-alpha-induced psoriasis.$^{68}$ Most cases of anti-TNF-alpha-induced psoriasis are linked to infliximab, with fewer cases described with adalimumab, certolizumab, and etanercept. ${ }^{67}$

The mechanisms underlying "paradoxical" psoriasis due to TNF-alpha antagonists are incompletely understood. The proinflammatory cytokine interferon-alpha produced by plasmacytoid dendritic cells might be implicated. ${ }^{64,69}$

Most patients with anti-TNF-alpha-induced psoriasis can be managed with topical corticosteroids, but switching to a different TNF-alpha blocker or to a biologic with a different treatment target may prove helpful. ${ }^{70}$ However, in $\sim 50 \%$ of the cases, the psoriasis will recur following treatment switch. ${ }^{66}$

\section{Anti-PDI drugs}

The anti-PD1 drugs nivolumab and pembrolizumab are immune checkpoint inhibitors that are used in oncology. Recognized side effects include immune-mediated toxicity, such as the development of psoriasis. ${ }^{71}$ Both psoriasis exacerbation and induction of de novo psoriasis have been reported. ${ }^{72}$

\section{Other monoclonal antibodies}

There have been several case reports reported on anecdotal associations of psoriasis with specific monoclonal antibodies. Examples include vedolizumab ( $\alpha 4 \beta 7$-integrin antagonist $)^{73}$ and rituximab (CD20 antagonist). ${ }^{74-77}$

\section{VEGF antagonists}

VEGF antagonists such as sorafenib, a small molecule inhibitor, have been reported in case reports and case series of psoriasis induction and exacerbation. Yiu et al ${ }^{78}$ reviewed three cases of sorafenib-induced psoriasis and four cases of sorafenib-exacerbated psoriasis. The association between sorafenib and psoriasis is considered paradoxal, given that VEFG inhibition is a potential treatment target for psoriasis. ${ }^{79}$ There have been several clinical cases reporting of complete psoriasis clearance in oncological patients treated with sorafenib. ${ }^{79,80}$

\section{Bupropion}

Bupropion is a nicotin receptor antagonist that is prescribed in smoking cessation aid. There have been several recent case reports on bupropion-associated psoriasis and cases of erythrodermic pustular psoriasis requiring in-hospital treatment. . $^{24,81-83}$

\section{Conclusion}

Exposure to certain medications can either induce or exacerbate psoriasis. However, the associations between specific drugs and psoriasis development have not been studied systematically. The available evidence is limited predominantly to anecdotal single-case reports or retrospective case series. Traditionally, drugs that are strongly implicated in psoriasis include beta-blockers, lithium, synthetic antimalarial drugs, and imiquimod. More recent associations have been reported for targeted treatments using monoclonal antibodies or small molecules that are used in oncology and immunology. Examples include TNF-alpha inhibitors such as infliximab and anti-PD1 immune check point inhibitors such as nivolumab. Clinicians should be aware of potential associations, but currently a practice gap exists in the recognition and management of drug-related psoriasis. The first step in resolving a drug-related case of psoriasis is recognizing a potential offending drug from the patients' medication list. Using the Naranjo adverse drug reaction probability scale may further help in the quantification of a potential link. Suspected medications may be discontinued and switched to an alternative medication when possible, while the psoriasis 
should be treated according to standard guidelines. Increased clinical awareness for medication-related causes of psoriasis induction or exacerbation should result in earlier recognition and treatment, which ultimately would lead to a better management in psoriasis treatment.

\section{Disclosure}

The authors report no conflicts of interest in this work.

\section{References}

1. Boehncke WH, Schon MP. Psoriasis. Lancet. 2015;386(9997):983-994.

2. Michalek IM, Loring B, John SM. A systematic review of worldwide epidemiology of psoriasis. J Eur Acad Dermatol Venereol. 2017;31(2):205-212.

3. Lowes MA, Suarez-Farinas M, Krueger JG. Immunology of psoriasis. Annu Rev Immunol. 2014;32:227-255.

4. Dika E, Bardazzi F, Balestri R, Maibach HI. Environmental factors and psoriasis. Curr Probl Dermatol. 2007;35:118-135.

5. Kim GK, Del Rosso JQ. Drug-provoked psoriasis: is it drug induced or drug aggravated? Understanding pathophysiology and clinical relevance. J Clin Aesthet Dermatol. 2010;3:32-38.

6. Tsankov N, Angelova I, Kazandjieva J. Drug-induced psoriasis. Recognition and management. Am J Clin Dermatol. 2000;1(3):159-165.

7. Abel EA, DiCicco LM, Orenberg EK, Fraki JE, Farber EM. Drugs in exacerbation of psoriasis. J Am Acad Dermatol. 1986;15(5 pt 1): 1007-1022.

8. Mrowietz U, Domm S. Systemic steroids in the treatment of psoriasis: what is fact, what is fiction? JEur Acad Dermatol Venereol. 2013;27(8): 1022-1025.

9. Dowlatshahi EA, Hollestein LM, Herings RM, Nijsten T, Wakkee M. Increased overall drug utilization in patients with psoriasis: a casecontrol study based on Dutch general practitioner data. Br J Dermatol. 2017;176(3):634-642.

10. Yiu ZZ. Psoriasis and increased drug utilization: a true burden of psoriasis or potential surveillance bias of comorbidities? Br J Dermatol. 2017;176(3):566-567.

11. Armstrong AW. Psoriasis provoked or exacerbated by medications: identifying culprit drugs. JAMA Dermatol. 2014;150(9):963.

12. Basavaraj KH, Ashok NM, Rashmi R, Praveen TK. The role of drugs in the induction and/or exacerbation of psoriasis. Int J Dermatol. 2010;49:1351-1361.

13. Dika E, Varotti C, Bardazzi F, Maibach HI. Drug-induced psoriasis: an evidence-based overview and the introduction of psoriatic drug eruption probability score. Cutan Ocul Toxicol. 2006;25(1):1-11.

14. Rongioletti F, Fiorucci C, Parodi A. Psoriasis induced or aggravated by drugs. J Rheumatol Suppl. 2009;83:59-61.

15. Fry L, Baker BS. Triggering psoriasis: the role of infections and medications. Clin Dermatol. 2007;25(6):606-615.

16. Naranjo CA, Busto U, Sellers EM, et al. A method for estimating the probability of adverse drug reactions. Clin Pharmacol Ther. 1981;30(2):239-245.

17. O'Brien M, Koo J. The mechanism of lithium and beta-blocking agents in inducing and exacerbating psoriasis. J Drugs Dermatol. 2006;5(5):426-432.

18. Gilliet M, Conrad C, Geiges M, et al. Psoriasis triggered by toll-like receptor 7 agonist imiquimod in the presence of dermal plasmacytoid dendritic cell precursors. Arch Dermatol. 2004;140(12):1490-1495.

19. Doyon JB, Liu KJ, Berman RA. Metoprolol-induced total body erythroderma. J Gen Intern Med. 2017;32(2):221-222.

20. Nestle FO, Conrad C, Tun-Kyi A, et al. Plasmacytoid predendritic cells initiate psoriasis through interferon-alpha production. J Exp Med. 2005;202:135-143.
21. Afshar M, Martinez AD, Gallo RL, Hata TR. Induction and exacerbation of psoriasis with Interferon-alpha therapy for hepatitis $\mathrm{C}$ : a review and analysis of 36 cases. J Eur Acad Dermatol Venereol. 2013;27(6): 771-778.

22. Swindell WR, Johnston A, Carbajal S, et al. Genome-wide expression profiling of five mouse models identifies similarities and differences with human psoriasis. PLoS One. 2011;6(4):e18266.

23. Sehgal VN, Dogra S, Srivastava G, Aggarwal AK. Psoriasiform dermatoses. Indian J Dermatol Venereol Leprol. 2008;74(2):94-99.

24. Singh PA, Cassel KP, Moscati RM, Eckersley D. Acute generalized erythrodermic pustular psoriasis associated with bupropion/naltrexone (contrave(R)). J Emerg Med. 2017;52(4):e111-e113.

25. Justiniano H, Berlingeri-Ramos AC, Sanchez JL. Pattern analysis of drug-induced skin diseases. Am J Dermatopathol. 2008;30(4):352-369.

26. Tsutsumi R, Yoshida Y, Yamamoto O. Imiquimod-induced psoriatic erythroderma treated with infliximab. Acta Derm Venereol. 2017;97(2):279-280.

27. Nast A, Gisondi P, Ormerod AD, et al. European S3-Guidelines on the systemic treatment of Psoriasis vulgaris--Update 2015--Short version-EDF in cooperation with EADV and IPC. J Eur Acad Dermatol Venereol. 2015;29(12):2277-2294.

28. Stanford CW, Kollipara R, Melookaran AM, Hall JC. Palmoplantar pustular psoriasis following initiation of a beta-blocker: disease control with low-dose methotrexate. Cutis. 2014;94(3):153-155.

29. Waqar S, Sarkar PK. Exacerbation of psoriasis with beta-blocker therapy. CMAJ. 2009;181(1-2):60.

30. Glass LR, Nguyen M, Winn BJ, Schrier A. Timolol drops causing reversible psoriatic fingernail changes. JAMA Ophthalmol. 2013;131(9):1134.

31. Baggio R, Le Treut C, Darrieux L, Vareliette A, Safa G. Psoriasiform diaper rash possibly induced by oral propranolol in an 18-monthold girl with infantile hemangioma. Case Rep Dermatol. 2016;8(3): 369-373.

32. Brauchli YB, Jick SS, Curtin F, Meier CR. Association between betablockers, other antihypertensive drugs and psoriasis: population-based case-control study. Br J Dermatol. 2008;158(6):1299-1307.

33. Wu S, Han J, Li WQ, Qureshi AA. Hypertension, antihypertensive medication use, and risk of psoriasis. JAMA Dermatol. 2014;150(9):957-963.

34. Carter TN. The relationship of lithium carbonate to psoriasis. Psychosomatics. 1972;13(5):325-327.

35. Brauchli YB, Jick SS, Curtin F, Meier CR. Lithium, antipsychotics, and risk of psoriasis. J Clin Psychopharmacol. 2009;29(2):134-140.

36. Jafferany M. Lithium and psoriasis: what primary care and family physicians should know. Prim Care Companion J Clin Psychiatry. 2008;10(6):435-439.

37. Damstra RJ, van Vloten WA. [Exacerbation of psoriasis caused by malaria prophylaxis with chloroquine and proguanil] Exacerbatie van psoriasis door malariaprofylaxe met chloroquine en proguanil. Ned Tijdschr Geneeskd. 1991;135(15):671-673.

38. Vestey JP, Savin JA. Psoriasis worsened by antimalarial prophylaxis. J Infect. 1992;24(2):211-212.

39. Gravani A, Gaitanis G, Zioga A, Bassukas ID. Synthetic antimalarial drugs and the triggering of psoriasis - do we need disease-specific guidelines for the management of patients with psoriasis at risk of malaria? Int J Dermatol. 2014;53:327-330.

40. Seminario-Vidal L, Hughey LS. Hydroxychloroquine de Novo-Induced Psoriasis in a patient with Lichen Planus Pigmentosus. Skinmed.2015;13(6): 492-494.

41. Vine JE, Hymes SR, Warner NB, Cohen PR. Pustular psoriasis induced by hydroxychloroquine: a case report and review of the literature. J Dermatol. 1996;23(5):357-361.

42. Friedman SJ. Pustular psoriasis associated with hydroxychloroquine. J Am Acad Dermatol. 1987;16(6):1256-1257.

43. Slagel GA, James WD. Plaquenil-induced erythroderma. J Am Acad Dermatol. 1985;12(5 pt 1):857-862.

44. Sorbara S, Cozzani E, Rebora A, Parodi A. Hydroxychloroquine in psoriasis: is it really harmful? Acta Derm Venereol. 2006;86(5):450-451. 
45. Smith WA, Siegel D, Lyon VB, Holland KE. Psoriasiform eruption and oral ulcerations as adverse effects of topical $5 \%$ imiquimod treatment in children: a report of four cases. Pediatr Dermatol. 2013;30(6):e157-e160.

46. Lemmenmeier E, Gaus B, Schmid P, Hoffmann M. A case of erythrodermia from exacerbated psoriasis vulgaris due to treatment of acute hepatitis C. BMC Dermatol. 2016;16(1):5.

47. Dag MS, Ozturk ZA, Yilmaz N, Cam H, Kadayifci A. Peginterferon alfa related psoriasis in a patient with acute hepatitis $\mathrm{C}$ and review of the literature. Wien Klin Wochenschr. 2013;125(17-18):537-540.

48. Tas F, Atsu N. Exacerbation of psoriasis induced by interferon-alpha treatment for melanoma. Cutan Ocul Toxicol. 2016;35(1):83-84.

49. Ladoyanni E, Nambi R. Psoriasis exacerbated by interferon-alpha in a patient with chronic myeloid leukemia. J Drugs Dermatol. 2005;4(2):221-222.

50. Besisik SK, Kocabey G, Caliskan Y. Major depression and psoriasis activation due to interferon-alpha in a patient with chronic myeloid leukemia; "overlooked and/or misdiagnosed adverse reaction in malignant disease". Am J Hematol. 2003;74(3):224.

51. Wolfer LU, Goerdt S, Schroder K, Zouboulis CC, Orfanos CE. [Interferon-alpha-induced psoriasis vulgaris] Interferon-alpha-induzierte Psoriasis vulgaris. Hautarzt. 1996;47(2):124-128.

52. Balak DM, Hengstman GJ, Cakmak A, Thio HB. Cutaneous adverse events associated with disease-modifying treatment in multiple sclerosis: a systematic review. Mult Scler. 2012;18(12):1705-1717.

53. Amschler K, Meyersburg D, Kitze B, Schon MP, Mossner R. Onset of psoriasis upon interferon beta treatment in a multiple sclerosis patient. Eur J Dermatol. 2016;26(2):211-212.

54. Lopez-Lerma I, Iranzo P, Herrero C. New-onset psoriasis in a patient treated with interferon beta-1a. Br J Dermatol. 2009;160(3):716-717.

55. Fierlbeck G, Rassner G, Muller C. Psoriasis induced at the injection site of recombinant interferon gamma. Results of immunohistologic investigations. Arch Dermatol. 1990;126(3):351-355.

56. Fierlbeck G, Rassner G. Treatment of psoriasis and psoriatic arthritis with interferon gamma. J Invest Dermatol. 1990;95(6 suppl):138S-141S.

57. Oda T, Sawada Y, Yamaguchi T, et al. Psoriatic erythroderma caused by terbinafine: a possible pathogenetic role for IL-23. J Investig Allergol Clin Immunol. 2017;27(1):63-64.

58. Mayser P. [Terbinafine: drug-induced lupus erythematodes and triggering of psoriatic skin lesions] Terbinafin: Medikamenteninduzierter Lupus erythematodes und Triggerung psoriatischer Hautveranderungen. Hautarzt. 2016;67:724-731.

59. Verros CD, Rallis E. The role of terbinafine in induction and/or exacerbation of psoriasis. Int J Dermatol. 2013;52:1155-1156.

60. Gupta AK, Sibbald RG, Knowles SR, Lynde CW, Shear NH. Terbinafine therapy may be associated with the development of psoriasis de novo or its exacerbation: four case reports and a review of drug-induced psoriasis. J Am Acad Dermatol. 1997;36:858-862.

61. Wilson NJ, Evans S. Severe pustular psoriasis provoked by oral terbinafine. Br J Dermatol. 1998;139(1):168.

62. Pauluzzi P, Boccucci N. Inverse psoriasis induced by terbinafine. Acta Derm Venereol. 1999;79(5):389.

63. Kim BS, Jang HS, Jwa SW, et al. Generalized pustular psoriasis and hepatic dysfunction associated with oral terbinafine therapy. $J$ Korean Med Sci. 2007;22:167-169.

64. Collamer AN, Battafarano DF. Psoriatic skin lesions induced by tumor necrosis factor antagonist therapy: clinical features and possible immunopathogenesis. Semin Arthritis Rheum. 2010;40(3):233-240.
65. Brown G, Wang E, Leon A, et al. Tumor necrosis factor-alpha inhibitorinduced psoriasis: systematic review of clinical features, histopathological findings, and management experience. J Am Acad Dermatol. 2017;76(2):334-341.

66. Guerra I, Perez-Jeldres T, Iborra M, et al. Incidence, clinical characteristics, and management of psoriasis induced by anti-TNF therapy in patients with inflammatory bowel disease: a nationwide cohort study. Inflamm Bowel Dis. 2016;22(4):894-901.

67. Olteanu R, Zota A. Paradoxical reactions induced by tumor necrosis factor-alpha antagonists: a literature review based on 46 cases. Indian $J$ Dermatol Venereol Leprol. 2016;82(1):7-12.

68. Pugliese D, Guidi L, Ferraro PM, et al. Paradoxical psoriasis in a large cohort of patients with inflammatory bowel disease receiving treatment with anti-TNF alpha: 5-year follow-up study. Aliment Pharmacol Ther. 2015;42(7):880-888.

69. Geller L, Kellen R. Tumor necrosis factor antagonist-induced psoriasis in a 3-year-old boy with Kawasaki disease. Dermatol Online J. 2017;23(2).

70. Faillace C, Duarte GV, Cunha RS, de Carvalho JF. Severe infliximabinduced psoriasis treated with adalimumab switching. Int J Dermatol. 2013;52(2):234-238.

71. Sibaud V, Meyer N, Lamant L, Vigarios E, Mazieres J, Delord JP. Dermatologic complications of anti-PD-1/PD-L1 immune checkpoint antibodies. Curr Opin Oncol. 2016;28(4):254-263.

72. Bonigen J, Raynaud-Donzel C, Hureaux J, et al. Anti-PD1-induced psoriasis: a study of 21 patients. J Eur Acad Dermatol Venereol. 2017;31(5):e254-e257.

73. Sody E, Korber A. Psoriasis induced by vedolizumab. Inflamm Bowel Dis. 2017;23(2):E9-E11.

74. Kim DW, Park SK, Woo SH, Yun SK, Kim HU, Park J. New-onset psoriasis induced by rituximab therapy for non-Hodgkin lymphoma in a child. Eur J Dermatol. 2016;26(2):190-191.

75. Venables ZC, Swart SS, Soon CS. Palmoplantar pustulosis secondary to rituximab: a case report and literature review. Clin Exp Dermatol. 2015;40(4):451-452.

76. Fiorillo L, Wang C, Hemmati I. Rituximab induced psoriasis in an infant. Pediatr Dermatol. 2014;31(6):e149-e151.

77. Guidelli GM, Fioravanti A, Rubegni P, Feci L. Induced psoriasis after rituximab therapy for rheumatoid arthritis: a case report and review of the literature. Rheumatol Int. 2013;33(11):2927-2930.

78. Yiu ZZ, Ali FR, Griffiths CE. Paradoxical exacerbation of chronic plaque psoriasis by sorafenib. Clin Exp Dermatol. 2016;41(4):407-409.

79. Crawshaw AA, Griffiths CE, Young HS. Investigational VEGF antagonists for psoriasis. Expert Opin Investig Drugs. 2012;21(1):33-43.

80. Antoniou EA, Koutsounas I, Damaskos C, Koutsounas S. Remission of psoriasis in a patient with hepatocellular carcinoma treated with sorafenib. In Vivo. 2016;30(5):677-680.

81. Matos-Pires E, Campos S, Mendes-Bastos P, Joao A, Fernandes C. Erythrodermic psoriasis induced by bupropion. $J$ Eur Acad Dermatol Venereol. 2017;31(2):e129-e130.

82. Surovik J, Riddel C, Chon SY. A case of bupropion-induced StevensJohnson syndrome with acute psoriatic exacerbation. J Drugs Dermatol. 2010;9:1010-1012.

83. Cox NH, Gordon PM, Dodd H. Generalized pustular and erythrodermic psoriasis associated with bupropion treatment. Br J Dermatol. 2002;146(6):1061-1063.
Psoriasis: Targets and Therapy

\section{Publish your work in this journal}

Psoriasis: Targets and Therapy is international, peer-reviewed, open access journal focusing on psoriasis, nail psoriasis, psoriatic arthritis and related conditions, identification of therapeutic targets and the optimal use of integrated treatment interventions to achieve improved outcomes and quality of life. Visit http://www.dovepress.com/testimonials.php to read real quotes from published authors. 